Bol. Acad. peru. leng. 66. 2019 (265-271)

\title{
EL CONDE DE LEMOS Y LA FRAGILIDAD ANIMAL DEL SIGLO XX
}

\author{
Allison Jazmín Domínguez Fernández \\ Colegio Mayor Secundario Presidente del Perú (COAR, Lima)
}

Fecha de recepción:

$18 / 10 / 2019$

Fecha de aceptación:

$30 / 11 / 2019$

Nacido en Ica el vigésimo séptimo día de abril de 1888, Abraham Valdelomar, llamado también Conde de Lemos, debido a que ese era el seudónimo que acogió en los años en que trabajó como redactor en el diario La Prensa, es considerado por muchos uno de los mejores escritores que nuestro país ha tenido. Desde su adolescencia, Valdelomar albergó el apego hacia la literatura, y este hecho queda demostrado en la creación de la revista La Idea Guadalupana cuando aún se hallaba en la secundaria. Por otro lado, gran parte de su vida se ha visto fuertemente influenciada por problemáticas que en pleno siglo xx eran completamente ignoradas y dejadas de lado por la sociedad de aquella época y que Valdelomar, por su parte - haciendo uso de su ingenio - ha logrado criticar sin herir la susceptibilidad del peruano del siglo pasado, usando sus escritos como sus principales medios de opinión. Asimismo, es reconocido principalmente por ser el autor de diversos textos tanto narrativos como periodís- 
ticos, que indican abiertamente el impetuoso daño que las costumbres adquiridas de los españoles, tales como las corridas de toros, las peleas de gallos o la venta de tortugas causaban en los animales y que sin duda demuestran una de las facetas más humanas de Valdelomar de la que muy pocos comentan: la de defensor de los animales. Por tales motivos, sostengo que Valdelomar, a través de sus obras, hizo lo posible por exponer el maltrato animal como una realidad social negativa que carecía de un fundamento sólido que la justificara y que, a pesar de considerarse "práctica cultural», atentaba contra la integridad física de los animales. En los párrafos siguientes, se mostrarán los argumentos que respaldan esta tesis.

En primer lugar, muchos años atrás el mismo Abraham ya habría expresado públicamente su postura en contra del maltrato a los animales. Desde su punto de vista, admite que: «En la medida en que el hombre no puede engendrar a este animal debe respetarlo, de la misma manera que yo respeto La divina comedia, porque comprendo que no podré escribirla» (1915). En el momento en que esta frase fue mencionada por el escritor, él se refería específicamente a los toros al decir «este animal», debido a que dicho comentario fue aludido en su crónica Los Toros; sin embargo, esto no quiere decir que no tuviera preocupación alguna por otras especies animales, ya que, como en los próximos argumentos se explicará, él también sentía respeto por otras criaturas, por lo que, en resumen, esta frase podría fácilmente aplicarse en contextos más generales. Por otro lado, al establecer una relación entre un libro magnífico y los animales en lo concerniente a su creación, pretende validar el hecho de que no se puede dejar de respetar la creación misma, que no es la propia. Con esta sencilla, pero contundente frase podemos fácilmente abrirnos paso entre los pensamientos sostenidos por Valdelomar, los cuales manifiestan el sentir del escritor peruano respecto de la ideología mantenida hace más de un siglo, la cual ensalza aquellas costumbres heredadas de los españoles — sin que importe el hecho de poner en riesgo la vida animal-, así como el pensamiento erróneo de las personas que consideran que nosotros somos y debemos creernos superiores ante cualquier otra especie por únicamente tener la condición de seres humanos. Frente a esto, Valdelomar se muestra 
https://doi.org/10.46744/bapl.201902.011

firme en su concepción del deber que tenemos cada uno de los seres humanos de respetar y preservar la fauna en su totalidad. Debido a estos razonamientos, muchos piensan que el escritor estaba adelantándose a su época al exponer una problemática que por aquellos años no era considerada como tal, pero, al transcurrir los años, nos damos cuenta de que, después de todo, Valdelomar tenía razón; ya que las leyes vigentes en la actualidad, que se encuentran en favor de la vida animal, respaldan su punto de vista.

Por otro lado, existen quienes afirman que los trabajos de Valdelomar no pudieron haberse tratado de una crítica a las costumbres de la época y que van en contra del bienestar animal, ya que la sociedad peruana del siglo xx no veía de forma errónea actos como las peleas de gallos o las corridas de toros y, por lo tanto, Valdelomar, al criarse en medio de esa sociedad, no podía ser la excepción. No obstante, esa concepción es totalmente absurda, ya que, independientemente de las medidas o pensamientos socialmente aceptados en dicha época, es justamente por criarse en medio de una sociedad que vulnera los derechos de los animales que Valdelomar pudo rechazarla y actuar abiertamente de acuerdo con su pensamiento, porque, como dice el dicho, solo se critica lo que se conoce, y aunque ciertamente existían influencias severamente marcadas en aquella época, la decisión final siempre la tuvo él mismo y tal como el periodista y estudioso de Abraham Valdelomar; Víctor Manriquez afirma: «Para él, los animales no solo son instinto, sino que poseen afectos y conciencia, quieren y sufren como los humanos» (2014). En otras palabras, en contracorriente con el pensamiento de los años en los que Valdelomar vivió, él ya tenía una ideología formada a partir del dolor que muchas personas que interiorizaron las costumbres «culturales» de la época, le producían a los animales, como se da por ejemplo en el caso de las corridas de toros, que por aquellos años eran muy populares como medios de diversión, ya que eran consideradas una tradición heredada de los españoles. Estas actividades culminaban con la muerte agónica del animal, punto que caló en los pensamientos del escritor y que contribuyó con la formación de sus ideales, a pesar de que dicha concepción no era considerada un problema por aquellos años. 
$\mathrm{Y}$ en relación con las corridas de toros, como antes ya se ha comentado, Valdelomar también manifestó su desaprobación en la crónica Los Toros. En dicho escrito, el autor se atrevió a criticar una popular costumbre limeña que por aquel entonces era aprobada y aceptada como un vestigio de nuestra herencia española; esta acción probablemente fue un indicio del sinsentido que este escritor encontraba a las festividades taurinas. De acuerdo con lo antes señalado, podemos destacar una frase indicada por él mismo: «Es un sutil calco del circo romano. Miles de personas reunidas en una plaza para ver sacrificados ya no seres humanos, sino toros» (1915), expresado de esa forma, se evidencia claramente el desprecio que Valdelomar tenía hacia esta actividad, considerada por muchos como cultural. Y, para reforzar esta idea, cabe resaltar el pensamiento del periodista Víctor Manriquez: «Él, en solitario, levanta la voz frente a lo que muchos defienden como cultura. Este pronunciamiento sea tal vez el primer esfuerzo en el Perú por crear conciencia en relación a un tema como la crítica a la tauromaquia» (2014); en otras palabras, lo que Manriquez desea transmitir es la idea de que el escritor iqueño fue uno de los primeros ciudadanos peruanos en reaccionar frente a un sufrimiento del que muchos eran indiferentes y que, muy por el contrario, veían el asesinato de toros como un medio de diversión y no como un atentado contra una forma de vida, por lo que, lejos de detenerlo, lo apoyaban al considerarlo parte de la cultura peruana. De la misma manera, Valdelomar, en otro fragmento de su crónica, repugna la tauromaquia con el siguiente argumento: «El toro, como el hombre, tiene conciencia, tiene sensibilidad y afectos» (1915); aquí el escritor se encarga de personificar a la criatura y señalar que como todo ser humano, este animal también siente y es consciente de las situaciones a las que se ve expuesto, volviendo de esta una postura que le ayuda a reforzar su idea de rechazo hacia esta actividad.

Asimismo, «El Caballero Carmelo» es un cuento considerado como una crítica a las peleas de gallo típicas de la época. Valdelomar lo deja muy en claro en su cuento, resaltando los sentimientos del narrador, y cómo este interpretaba los sentimientos de Carmelo a través de sus acciones. En una de las cartas de Valdelomar a su madre, este afirma que su cuento fue escrito con alusiones a su niñez en Pisco (s.f.) y, al ser un 
https://doi.org/10.46744/bapl.201902.011

cuento con temática triste y constantes reclamos en contra de las peleas de gallos, nos damos cuenta de que su infancia sí estuvo íntimamente relacionada con la creación de sus escritos relacionados. En el texto mismo (1913) se señala: «[...] El Carmelo iría a un combate y a luchar a muerte, $[\ldots]$ con un gallo más fuerte y más joven. Hacía ya tres años que estaba en casa, había él envejecido mientras crecíamos nosotros. ¿Por qué aquella crueldad de hacerlo pelear? [...]» (p. 8) El narrador se encarga de personificar al Carmelo, dotándolo de cualidades humanas y atribuyéndole acciones y características que se asemejan a las realizadas por el hombre, tales como la lucha en un combate o el envejecimiento, por tales motivos produce en el lector un sentimiento de identificación con el animal al establecer puntos en común entre este y el hombre, provocando al mismo tiempo que un sentimiento de lástima hacia la criatura se desarrolle en la mente del lector. Además, este fragmento pone de manifiesto parte del pensamiento de Valdelomar, porque si bien es cierto que él no es el narrador de la obra, sí es el autor y, tal como lo indicó en su momento, sus experiencias personales fueron plasmadas en «El caballero Carmelo», brindándonos otra razón para creer que él era un defensor de los animales al mostrarse en contra de actos deplorables como las apuestas a costa de la vida de animales, que no han dañado a nadie, tal como el gallo Carmelo.

De igual manera, Valdelomar defiende otra forma de vida animal en La psicología de las tortugas. En este ensayo, se expone cómo el hombre se comporta de manera inhumana frente a una criatura que no le ha causado ningún daño, tal como Valdelomar señala: «En Ceilán las venden a pedazos, porque $[\ldots]$ quieren siempre la carne fresca, y como el corazón es lo menos agradable de la tortuga, las infelices viven días sucesivos sufriendo consecuentes mutilaciones hasta que un comprador pide el corazón. Entonces mueren» (1915). Este fragmento, además de exhibir una costumbre espeluznante del siglo pasado que atenta contra el bienestar de los animales y su protección, manifiesta también el rechazo de Valdelomar hacia estas prácticas de la vida cotidiana en aquel entonces, y de igual modo da a conocer la perspectiva de que una tortuga es una criatura de carácter pasivo que no representa ningún peligro para el ser humano, lo cual no brinda una justificación adecuada para aceptar 
https://doi.org/10.46744/bapl.201902.011

el comportamiento del peruano del siglo $\mathrm{xx}$, y esto era un aspecto que Valdelomar tenía en cuenta al momento de refutar dichas prácticas comerciales.

En síntesis, la vida y las experiencias de Abraham Valdelomar tuvieron un fuerte impacto en la elaboración de sus escritos, en donde se puso de manifiesto todo su pensamiento crítico y se expuso una perspectiva que no era compartida por la mayor parte de los peruanos contemporáneos a él. En este sentido, reafirmo la idea de que Valdelomar a través de su obra expone la problemática del maltrato animal como una «costumbre» que afecta directamente de forma negativa a estas criaturas y que no era considerada dañina por aquella época. De esta forma, logra manifestar su ideología basada en el cuidado y respeto hacia los animales, quizás gracias a una sensibilidad adelantada a su tiempo, pero que, actualmente, se ha convertido en ideas que están respaldadas por normas. Por tales motivos, claramente se ha demostrado que el iqueño tenía razón y, con suma facilidad, su forma de pensar pudo haber ejercido cierto grado de influencia al formular nuevas perspectivas en favor de criaturas indefensas como los animales maltratados, con el fin de lograr que el hombre entienda que su accionar errado cala de forma negativa en la integridad de estas especies. 
https://doi.org/10.46744/bapl.201902.011

\section{BIBLIOGRAFÍA}

LITERALGIA. (2018). Abraham Valdelomar: Un escritor que se oponía a las corridas de toros. 09-09-19, de literalgia. Sitio web: https://www.literalgia.com/abraham-valdelomar-unantitaurino-con-mucha-clase/ http://www.cartaabierta.pe/ valdelomar-defensor- los-animales/.

PERUEDUCA. (s. f.). El caballero Carmelo. 05-09-19, de PERÚEDUCA Sitio web: file:///C:/Users/20190294/Downloads/recursos primaria recursosedu relatos comunicacion caballerocarmelo. pdf.

HISTORIA DEL PERÚ. (s. f.). Abraham Valdelomar. 30-0819, de HISTORIA DEL PERÚ. Sitio web: https:// historiaperuana.pe/bioqrafia/abraham-valdelomar/.

UNMSM. (s. f.). Biografía de Abraham Valdelomar. 01-09-19, de UNIVERSIDAD NACIONAL MAYOR SAN MARCOS. Sitio web: http://www.unmsm.edu.pe/ilustres/biografia/75. 